\title{
Drag Reduction in Turbulent Boundary Layers with Half Wave Wall Oscillations
}

\author{
Maneesh Mishra and Martin Skote \\ School of Mechanical \& Aerospace Engineering, Nanyang Technological University, 50 Nanyang Avenue, Singapore 639798 \\ Correspondence should be addressed to Martin Skote; mskote@ntu.edu.sg
}

Received 29 August 2014; Revised 17 October 2014; Accepted 17 October 2014

Academic Editor: Kim M. Liew

Copyright ( 2015 M. Mishra and M. Skote. This is an open access article distributed under the Creative Commons Attribution License, which permits unrestricted use, distribution, and reproduction in any medium, provided the original work is properly cited.

\begin{abstract}
Spatial square waves with positive cycle are used as steady forcing technique to study drag reduction effects on a turbulent boundary layer flow. Pseudospectral method is used for performing direct numerical simulations on very high resolution grids. A smooth step function is employed to prevent Gibbs phenomenon at the sharp discontinuities of a square wave. The idea behind keeping only the positive cycle of the spatial forcing is to reduce the power consumption to boost net power savings. For some spatial frequency of the oscillations with half waves, it is possible to prevent recovery of skin friction back to the reference case values. A set of wall oscillation parameters is numerically simulated to study its effect on the power budget.
\end{abstract}

\section{Introduction}

Turbulent drag reduction is one of the active areas of research in fluid mechanics especially due to its global impact on sustainability challenges we are facing today. One of the active techniques for reducing drag is through spanwise oscillation of the wall which reduces the skin friction and promises to have large potential for energy savings.

This curious phenomenon was first observed by Jung et al. [1] through direct numerical simulations (DNS) in a channel flow. Since then, a lot of research efforts have been made in this direction for different flow configurations like channel flow [2-9], pipe flow [10, 11], and boundary layer flows [12-14]. These investigations have been made either through experiments or using DNS.

Most of these studies have dealt with temporal form of wall oscillations which are specified as

$$
w(t)_{y=0}=W_{m} \sin (\omega t),
$$

where $W_{m}$ is the amplitude and $\omega$ is the frequency of the imposed oscillations.

A few studies have been devoted to explore the spatial oscillations and its impact on reducing skin friction has been found to be greater than for temporal oscillations [15-19].
Spatial oscillations can be realized by enforcing the following boundary condition:

$$
w(x)_{y=0}=W_{m} \sin (k x),
$$

where $k$ is the spatial frequency of oscillation and is related to the wavelength $(\lambda)$ as $k=2 \pi / \lambda$.

Spatial wall oscillation technique has its advantages and disadvantages. It can have greater drag reduction as compared to temporal oscillations and, hence, there are higher net energy savings. It is an open-loop method so we do not require an array of distributed sensors on the surface. However, the implementation remains a challenge as it requires numerous moving parts which makes it impractical. Although there have been advances in the field of material science research to realize such waveforms in practical situations, the physical realization still remains elusive with the current technology.

Almost all of the previous works have implemented oscillation waveforms using sinusoidal functions. A recent study by Cimarelli et al. [20] explored different temporal waveforms. In this work, we would like to explore the possibility of using spatial square waves for drag reduction. In order to reduce the power required to incorporate these oscillations, we consider only the positive cycles of these oscillations. One of the ways to realize spatial oscillations can be via pulsed jets 


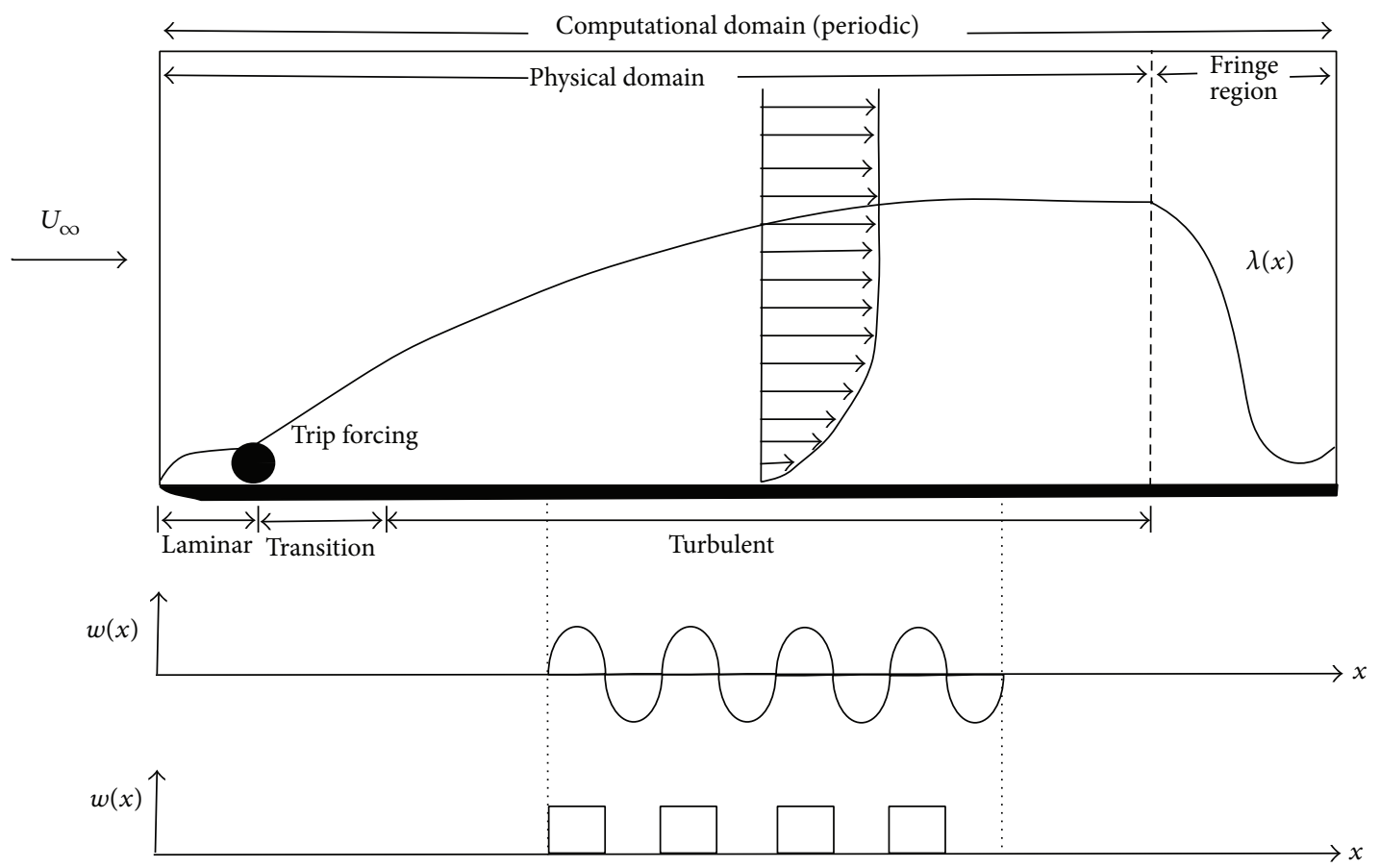

FIGURE 1: Computational box as seen from the negative $z$-direction with the growth of boundary layer illustrated. Fringe region forces the solution back to the prescribed laminar inflow thus enforcing periodic boundary condition. The lower part illustrates the spanwise velocity forcing which is applied in a part of the wall under the turbulent boundary layer. Half square waves are used in the present study as shown in the bottom figure.

in the spanwise direction for the near wall region. Another possibility is to actively manipulate wall roughness optimally distributed along the surface. The main contribution of the present work is to illustrate the use of smooth step functions to approximate the square waves which otherwise may give rise to Gibbs phenomenon when using spectral methods.

\section{Methodology}

2.1. Governing Equations. The governing equations which are used for the simulations here are the Navier-Stokes equations which are formulated in terms of velocity-vorticity and written in tensor notation as

$$
\begin{gathered}
\frac{\partial u_{i}}{\partial t}=-\frac{\partial p}{\partial x_{i}}+\epsilon_{i j k} u_{j} \omega_{k}-\frac{\partial}{\partial x_{i}}\left(\frac{1}{2} u_{j} u_{j}\right)+\frac{1}{\operatorname{Re}} \nabla^{2} u_{i}+F_{i} \\
\frac{\partial u_{i}}{\partial x_{i}}=0
\end{gathered}
$$

where $u_{i}$ are the velocity components, $\omega_{i}$ are the vorticity components, $p$ denotes pressure, and $F_{i}$ is the body force. The nondimensional constant $\operatorname{Re}=U_{\infty} \delta^{*} / \nu$ is the Reynolds number with $U_{\infty}$ being the streamwise freestream velocity, $\delta^{*}$ is the displacement thickness at $x=0$, and $\nu$ is the kinematic viscosity. $x_{i}$ represents the coordinate system with $(x, y, z)$ as streamwise, wall-normal, and spanwise coordinates and $t$ denotes time.

These equations are solved using a pseudospectral method with appropriate boundary conditions. The basic idea with spectral methods is to express the solution as a sum of basis functions and then compute their coefficients such that they satisfy the governing partial differential equations and the boundary conditions.

A third-order Runge-Kutta-scheme is used to perform time integration for the nonlinear terms. A second-order Crank-Nicolson method is used for the linear terms. For removing aliasing errors, a 3/2-rule is applied to the evaluation of the nonlinear terms when calculating Fourier transforms in the wall parallel $(x-z)$ plane. The numerical code (SIMSON [21]) used for the simulations in this work has been developed at KTH, Stockholm. Earlier simulations of both temporal $[12,13]$ and spatial $[15,16]$ wall forcing have been performed with the code.

2.2. Numerical Setup. Since we are trying to simulate a turbulent boundary layer with a spatially growing boundary layer, we need to choose our basis functions accordingly. A basic sketch for the computational setup is shown in Figure 1. For the discretization in the streamwise-spanwise plane, Fourier basis is chosen assuming the solutions are periodic in these directions. However, for the wall normal direction, periodicity does not apply and Chebyshev polynomials are instead used as basis functions for the $y$ (wallnormal) direction. The technique is similar to other spectral codes used for channel flows $[5-8,17,18]$, and the spectral accuracy is a considerable advantage as compared to other discretizations, such as the finite volume methods used in, for example, $[9,14]$. 


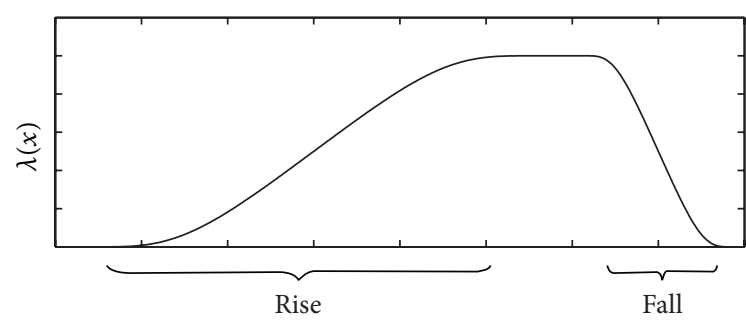

FIGURE 2: Schematic picture of the fringe region.

For initializing the simulations, a laminar base flow is required and is given as the Blasius similarity solution [22]. A trip forcing using a random volume force is then incorporated at $x=5$ for the flow to undergo transition and thereafter we have turbulent flow regime. Figure 1 depicts this scheme.

Particular attention must be given to the streamwise direction since the boundary layer is growing downstream and hence no natural periodicity exists in that direction. Therefore, for the purpose of artificially creating a periodic computational domain, a fringe region is introduced at the end to achieve this. The purpose of this fringe region is to dampen the velocity fluctuations to zero and bring the velocity field back to the laminar Blasius solution such that there are minimum upstream effects [23]. This is achieved by introducing the volume forcing $F_{i}$ in (3):

$$
F_{i}=\lambda(x)\left(\tilde{u}_{i}-u_{i}\right)
$$

where $\lambda(x)$ is the strength of the forcing and $\widetilde{u}_{i}$ is the laminar inflow velocity profile. The function $\lambda$ is defined as

$$
\lambda(x)=\lambda_{\text {max }} f(x)
$$

with

$$
f(x)=S\left(\frac{x-x_{\text {start }}}{\Delta x_{\text {rise }}}\right)-S\left(\frac{x-x_{\text {end }}}{\Delta x_{\text {fall }}}+1\right) .
$$

Here $\lambda_{\max }$ is the maximum strength of the fringe, $x_{\text {start }}$ and $x_{\text {end }}$ denote the spatial extent of the region where the fringe is nonzero, and $\Delta x_{\text {rise }}$ and $\Delta x_{\text {fall }}$ are the rise and fall distance of the fringe function, respectively. Figure 2 shows a schematic of how the fringe function varies. $S(\eta)$ is a continuous step function that varies from zero for $\eta \leq 0$ to unity for $\eta \geq 1$ and is given by

$$
S(\eta)= \begin{cases}0, & \eta \leq 0, \\ \frac{1}{\left(1+e^{(1 /(\eta-1)+1 / \eta)}\right)}, & 0<\eta<1, \\ 1, & \eta \geq 1 .\end{cases}
$$

2.3. Wall Oscillation Implementation. The form of wall oscillation implemented here is a spatial square wave with only positive forcing to reduce power consumption. However, there are numerical challenges in implementing this using pseudospectral method. A square wave when represented using Fourier basis gives rise to Gibbs phenomenon which
TABLE 1: Oscillation parameters for the simulations presented.

\begin{tabular}{lcc}
\hline Parameter set (PS) & $W_{m}$ & $k$ \\
\hline PS1 & $(0.1,0.25,0.4,0.5,0.6,0.75,1)$ & 0.0628 \\
PS2 & $(0.1,0.25,0.4,0.5,0.6,0.75,1)$ & 0.1256 \\
PS3 & $(0.1,0.25,0.4,0.5,0.6,0.75,1)$ & 0.2512 \\
\hline
\end{tabular}

is shown in Figure 3(a). When we try to approximate the strong discontinuity in the square wave, it results in strong oscillations at the edges. These result in spurious values causing numerical instability and large computational errors. Increasing the number of terms in the Fourier series approximation does reduce the oscillation but it does not eliminate it completely.

In order to avoid Gibbs phenomenon, we utilize the same step function as we used for fringe region (8). Using the step function is advantageous as it has continuous derivatives at all points and does not exhibit the spurious ringing phenomenon. Figure 3(b) shows the use of $f(x)$ in implementing the wall boundary condition for the present simulations. By including only a few Fourier coefficients, we can approximate the function quite accurately and eliminate Gibbs rings.

Spatial wall oscillation can be incorporated with the following boundary condition:

$$
w(x)_{y=0}=W_{m} f(x),
$$

where $f(x)$ is the same profile function as used for fringe region (see equation (7)) and $W_{m}$ is the amplitude of the spatial oscillations.

2.4. Numerical Parameters. All quantities are nondimensionalized by the free-stream velocity $\left(U_{\infty}\right)$ and the displacement thickness $\left(\delta^{*}\right)$ at the starting position of the simulation $(x=$ 0 ), where the flow is laminar. The Reynolds number is set by specifying $\operatorname{Re}_{\delta^{*}}=U_{\infty} \delta^{*} / \nu$ at the laminar inlet $(x=0)$. Note that, unless otherwise stated, the + superscript indicates that the quantity is made nondimensional with the friction velocity of the unmanipulated boundary layer (the reference case), denoted by $u_{\tau}^{0}$, and the kinematic viscosity $(\nu)$.

A computational domain with $L_{x}=600, L_{y}=30$, and $L_{z}=34$ is chosen with a mesh resolution of $800 \times 201 \times 144$, respectively. The resolution of these simulations in wall units is $\Delta x^{+}=16, \Delta y_{\min }^{+}=0.04$, and $\Delta z^{+}=5.1$. All scalings are done based on $u_{\tau}^{0}$ from reference case at the starting position of wall forcing $(x=200)$. Wall oscillation boundary conditions are employed between $x_{\text {start }}=200$ and $x_{\text {end }}=450$ once it is ascertained that the flow has become fully turbulent. The Reynolds number based on momentum thickness varies between $450<\operatorname{Re}_{\Theta}<715$ in the control region.

Table 1 summarizes the parameters chosen for the steady spatial oscillation in the present work. Only the positive forcing has been employed for these simulation setups as shown in Figure 3(b). The spatial frequencies have been doubled and halved with respect to PS2 to see its impact on drag reduction performance. Also, the amplitude of oscillations is varied to understand its impact on drag reduction. The aim is to observe the effect of removing the negative forcing of the 


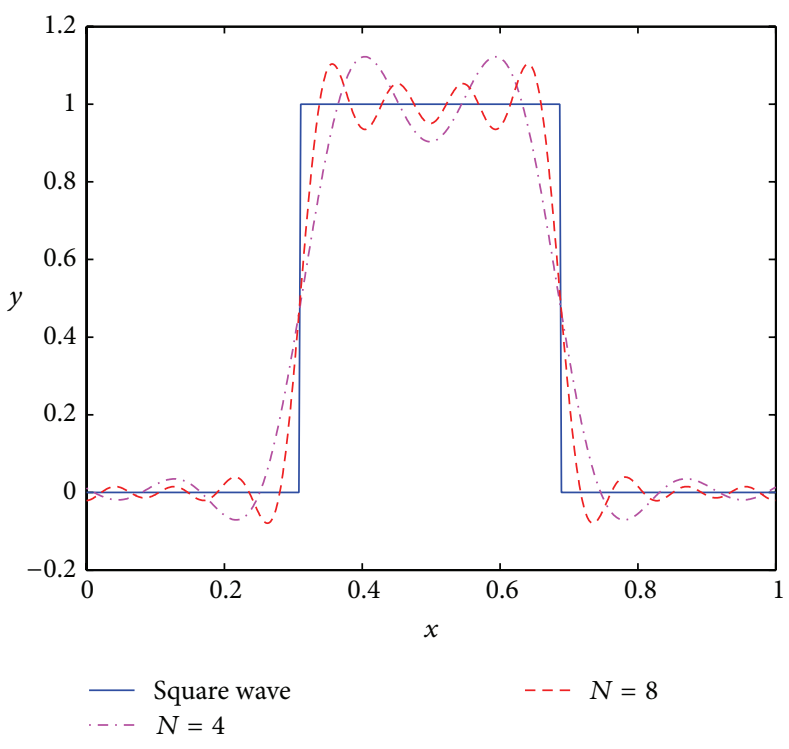

(a) Square wave with Gibbs phenomenon

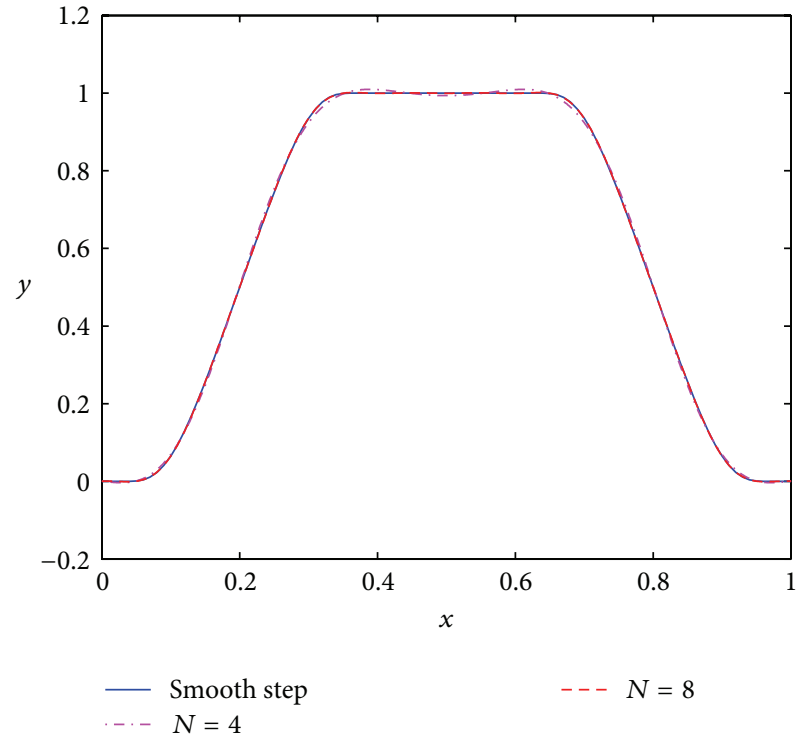

(b) Smooth step function

FiguRE 3: Function approximations using finite Fourier series terms.

PS1
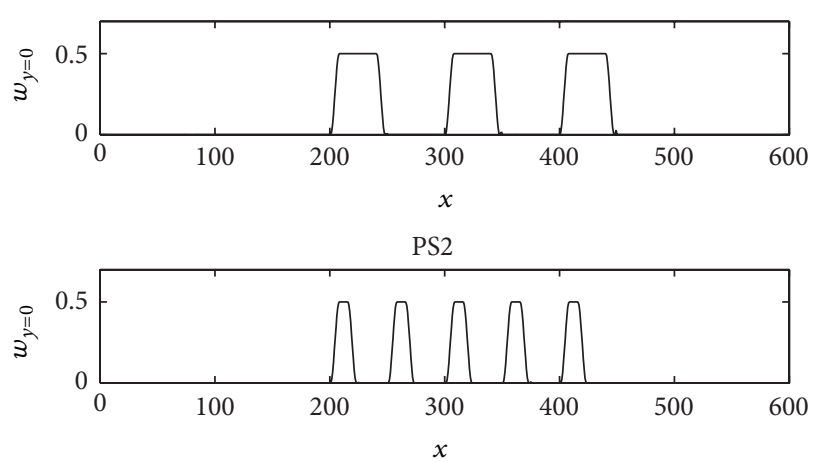

PS3

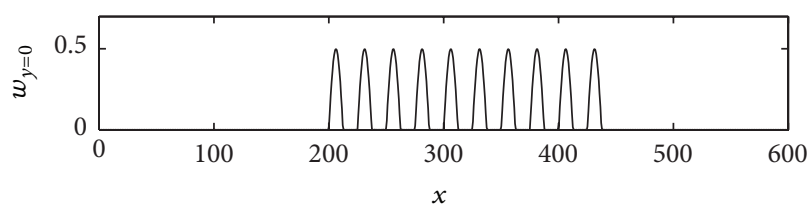

FIgURE 4: Wall boundary condition set for spanwise velocity component for PS1, PS2, and PS3 at $W_{m}=0.5$.

wall boundary and its effects on power budget and net energy savings. Figure 4 shows the wall boundary condition for the three parameter sets at $W_{m}=0.5$.

\section{Results and Discussion}

In this section, we look into two aspects of the results obtained from our numerical simulations. First, we look into attenuation of $c_{f}$ values with respect to the reference case.
Subsequently, we present the power budget based on the different forcings.

3.1. Skin Friction Attenuation. We compare skin friction from the unoscillated or the reference case with the oscillated cases. Skin friction coefficient for turbulent flows is defined as

$$
c_{f}=2\left(\frac{u_{\tau}}{U_{\infty}}\right)^{2},
$$

where $u_{\tau}$ is the friction velocity and is computed based on mean streamwise velocity gradient at the wall:

$$
u_{\tau}=\sqrt{\left.\nu \frac{\partial u}{\partial y}\right|_{y=0}} .
$$

The resulting drag reduction (DR) is then calculated from

$$
\operatorname{DR}(\%)=100 \frac{c_{f}^{0}-c_{f}}{c_{f}^{0}},
$$

where $c_{f}^{0}$ is the skin friction of the reference case. In contrast to internal flows [1-11], the DR is varying in the downstream direction for the present case of boundary layer flow. Figure 5 shows the results for the skin friction variation along streamwise direction. All three cases show skin friction attenuation. As soon as wall oscillation is applied at $x_{\text {start }}=200$, we see a strong gradient which marks the spatial transient for $c_{f}$. For PS1, we have a longer wavelength and, due to discontinuous half waves, we observe recovery of $c_{f}$ back towards the reference case. However, for PS2 and PS3, due to smaller wavelength, this recovery process is weaker. This is a crucial observation as it indicates that, with positive forcing itself, we can get drag reduction of a similar order of magnitude as with 


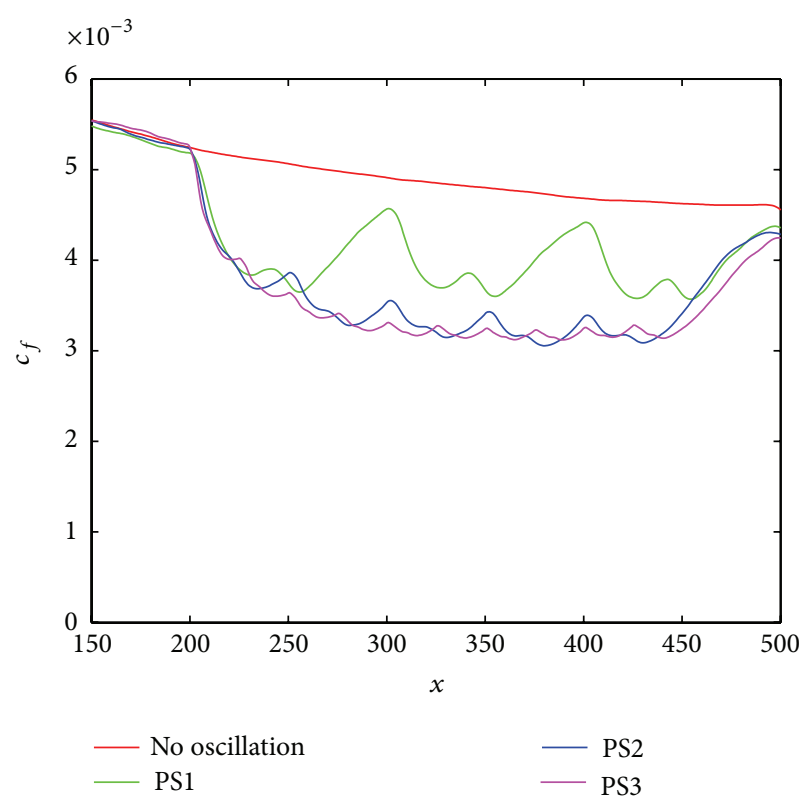

FIGURE 5: Spatial development of skin friction along streamwise direction at $W_{m}=1$.

a full cycle of wall oscillation. This would reduce the power required for forcing the wall oscillation and this increases our net power saving as will be presented in the next section. At $x_{\text {end }}=450$, the oscillations are stopped and skin friction attains the reference case values.

3.2. Power Budget. To compute the net energy savings, we need to take into account the energy required for wall oscillation as compared to the savings due to drag reduction. The derivation of these terms was given for channel flow by Quadrio and Ricco [6] which was extended to the boundary layer case by Skote [13].

In order to compute the saved power $P_{\text {sav }}(\%), D R$ (as percentage of ratio of skin-friction coefficients from reference and oscillated cases; see (12)) is integrated for the region with wall oscillation. The total saved power can be written as

$$
P_{\text {sav }}(\%)=\frac{1}{L} \int_{x_{\text {start }}}^{x_{\text {end }}} \mathrm{DR}(\%) \mathrm{d} x \text {, }
$$

where $x_{\text {start }}$ denotes the position at which the wall oscillation is started, $x_{\text {end }}$ denotes the endpoint for oscillation, and $L=$ $x_{\text {start }}-x_{\text {end }}$.

Similarly, the wall oscillation requires power input which can also be defined in terms of the friction power of the reference flow [13] and can be written as

$$
P_{\text {req }}(\%)=\frac{\left.\int_{x_{\text {start }}}^{x_{\text {end }}} \nu(\partial w / \partial y)\right|_{y=0} W \mathrm{~d} x}{\int_{x_{\text {start }}}^{x_{\text {end }}}\left(u_{\tau}^{0}\right)^{2} U_{\infty} \mathrm{d} x} .
$$

The net saved power is then defined as $P_{\text {net }}=P_{\text {sav }}-P_{\text {req }}$. If $P_{\text {net }}$ is negative it indicates that the input power required to oscillate the wall is greater than the saved power due to

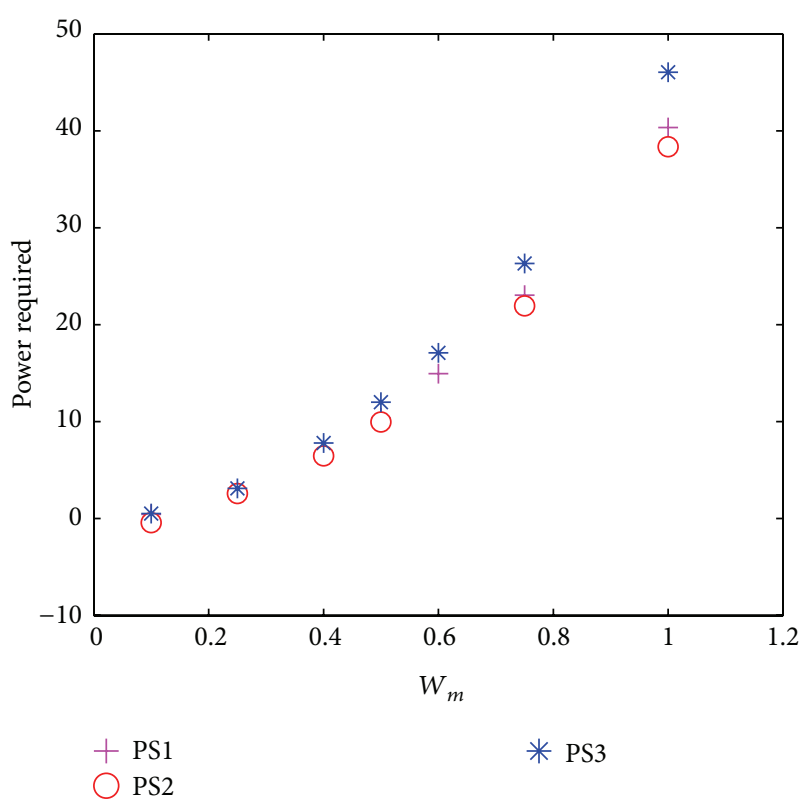

FIgURE 6: Power required for wall oscillation.

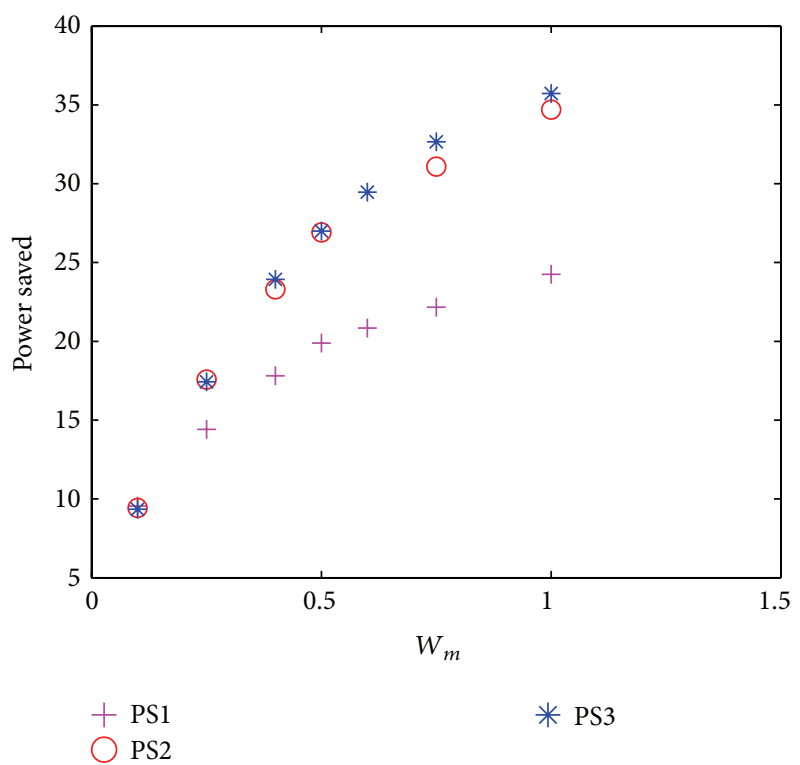

FIGURE 7: Power saved for the three chosen parameter sets.

streamwise drag reduction. However, it is possible that, for an optimized set of oscillation parameters, one may achieve positive energy budget. As reported by other researchers $[15,18,19]$, a positive net energy is more attainable for spatial forcing than for temporal forcing.

Power required for the three oscillation cases is shown in Figure 6. For lower amplitudes of forcing, we require lesser power and it grows exponentially for larger amplitudes. For different spatial frequencies, there is not much difference in input power required. Figure 7 shows the power saved based on (13). Here, we see that with increasing amplitude, the power saving saturates after a limit. The effect of spatial 


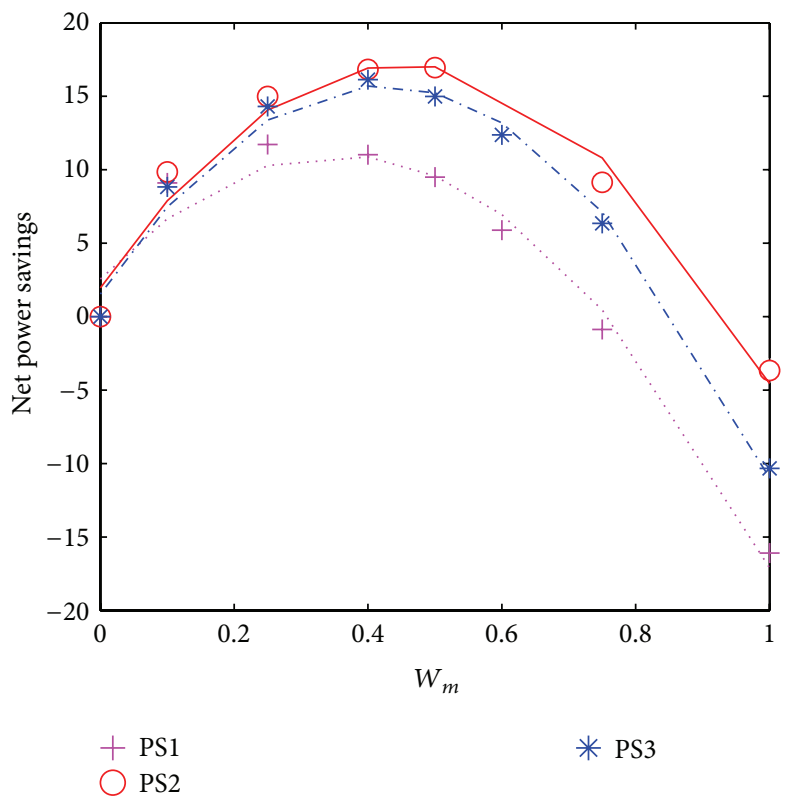

FIGURE 8: Net power savings for the three chosen parameter sets together with a quadratic curve fit.

frequency is rather interesting as we see that the power saved for PS2 and PS3 is almost the same. PS1 has a lower power saving and that can be attributed to the recovery to unoscillated skin friction values discussed previously in Section 3.1.

Figure 8 shows the net power savings for the different parameter sets. PS2 with $W_{m}=0.5$ gives us the maximum net power saving $(\sim 18 \%)$ amidst our chosen parameter space. Performance quickly deteriorates for larger amplitudes which show negative net power savings indicating that we spend more power in oscillating the wall as compared to the power savings. No complete description of the drag reduction mechanism exists to date. Thus, the influence of the parameters on the drag reduction remains largely unexplained. The parameter space explored in the current work is definitely not exhaustive. Viotti et al. [17] show power budget statistics for a wide parameter space using sinusoidal wall oscillation. The maximum net power savings achieved is reported as $23 \%$ which was found at $W_{m}^{+}=6$. On the other hand, they showed maximum net power savings of $6 \%$ at $W_{m}^{+}=12$, which is the amplitude comparable to the present case. They concluded that lower amplitudes give higher net power savings, even though the drag reduction values are lower. The simulation cases in the present study are not as exhaustive due to the computational demands for spatially developing boundary layers. Nevertheless, for the current study we obtain the maximum savings of $18 \%$ at $W_{m}^{+}=10$. The current suboptimal results may very well be further improved by increasing the parametric space.

Although the results look promising in terms of the net power savings, one of the drawbacks of the proposed methodology is that it induces crossflow which might be undesirable in certain situations. In order to illustrate the phenomena, a horizontal plane at $y^{+}=10$ is shown in Figure 9. The figure has been compressed by a factor 4 in the streamwise direction for better visualization. From the figure it is observed that, after the first oscillation stops, the streaks reorient themselves to the streamwise direction. However, a spanwise crossflow manifests itself as can be seen by the oblique streaks in the regions after the second and third periods of forcing.

Note that this phenomena would not occur if a periodic function with equal amount of positive and negative spanwise wall velocity is used, as in the study of temporal nonsinusoidal wall forcing by Cimarelli et al. [20].

\section{Conclusion}

A new form of steady spatial wall oscillation technique in the form of square waves with positive forcing has been presented with promising results for developing an active drag reduction technique. Spectral methods were used to solve the governing equations and the use of a smooth step has been demonstrated to approximate a square wave to overcome Gibbs phenomenon and avoid sharp discontinuities. Downstream development of skin friction and power budget for different oscillation parameters have been presented. An optimal set of wall oscillation parameters for the current parameter space was found to have $\sim 18 \%$ net energy savings.

\section{Conflict of Interests}

The authors declare that there is no conflict of interests regarding the publication of this paper. 


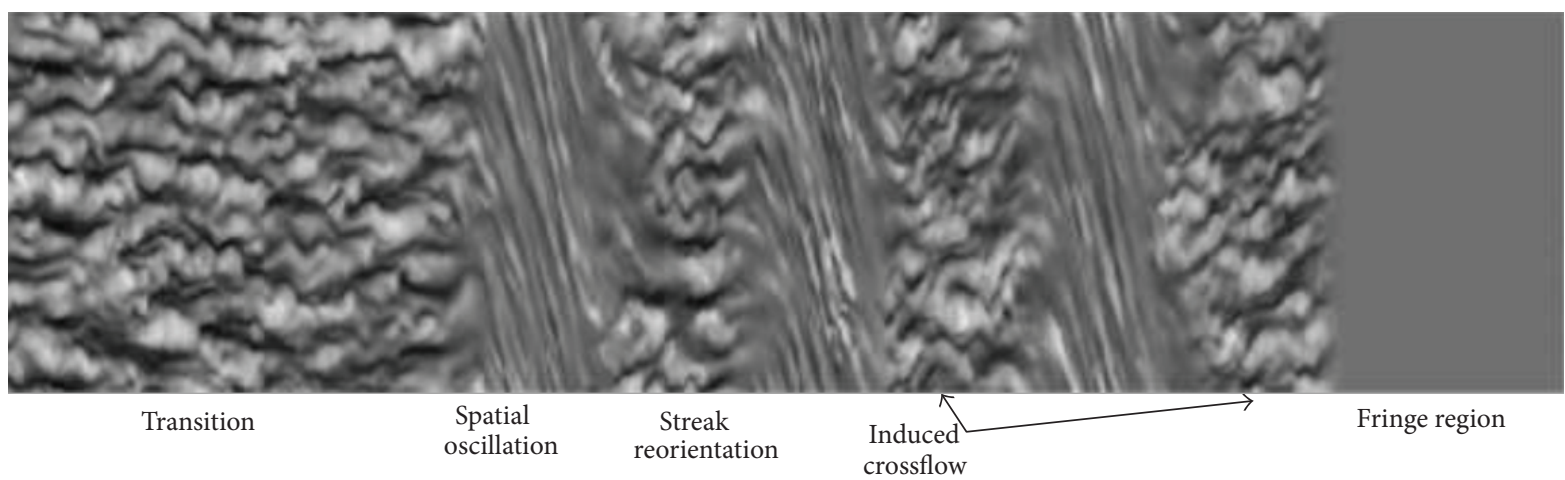

FIgURE 9: Half wave oscillations inducing crossflow in the spanwise direction. A horizontal plane $\left(y^{+}=10\right)$ in the computational box is shown. The dark grey indicates low-speed regions and lighter shades indicate high-speed patches. The plane is stretched by a factor of four in the spanwise direction for clarity.

\section{Acknowledgment}

This research work is supported in part by Singapore MOE Tier-2 Grant no. MOE2012-T2-1-030.

\section{References}

[1] W. J. Jung, N. Mangiavacchi, and R. Akhavan, "Suppression of turbulence in wall-bounded flows by high-frequency spanwise oscillations," Physics of Fluids A, vol. 4, no. 8, pp. 1605-1607, 1992.

[2] A. Baron and M. Quadrio, "Turbulent drag reduction by spanwise wall oscillations," Applied Scientific Research, vol. 55, no. 4, pp. 311-326, 1995.

[3] J.-I. Choi, C.-X. Xu, and H. J. Sung, "Drag reduction by spanwise wall oscillation in wall-bounded turbulent flows," AIAA Journal, vol. 40, no. 5, pp. 842-850, 2002.

[4] C.-X. Xu and W.-X. Huang, "Transient response of Reynolds stress transport to spanwise wall oscillation in a turbulent channel flow," Physics of Fluids, vol. 17, no. 1, Article ID 018101, 2005.

[5] M. Quadrio and P. Ricco, "Initial response of a turbulent channel flow to spanwise oscillation of the walls," Journal of Turbulence, vol. 4, article 7, 2003.

[6] M. Quadrio and P. Ricco, "Critical assessment of turbulent drag reduction through spanwise wall oscillations," Journal of Fluid Mechanics, vol. 521, pp. 251-271, 2004.

[7] P. Ricco and M. Quadrio, "Wall-oscillation conditions for drag reduction in turbulent channel flow," International Journal of Heat and Fluid Flow, vol. 29, no. 4, pp. 891-902, 2008.

[8] P. Ricco, C. Ottonelli, Y. Hasegawa, and M. Quadrio, "Changes in turbulent dissipation in a channel flow with oscillating walls," Journal of Fluid Mechanics, vol. 700, pp. 77-104, 2012.

[9] E. Touber and M. A. Leschziner, "Near-wall streak modification by spanwise oscillatory wall motion and drag-reduction mechanisms," Journal of Fluid Mechanics, vol. 693, pp. 150-200, 2012.

[10] A. Duggleby, K. S. Ball, and M. R. Paul, "The effect of spanwise wall oscillation on turbulent pipe flow sturctures resulting in drag reduction," Physics of Fluids, vol. 19, no. 12, Article ID 125107, 2007.

[11] F. Auteri, A. Baron, M. Belan, G. Campanardi, and M. Quadrio, "Experimental assessment of drag reduction by traveling waves in a turbulent pipe flow," Physics of Fluids, vol. 22, no. 11, Article ID 115103, 2010.

[12] I. Yudhistira and M. Skote, "Direct numerical simulation of a turbulent boundary layer over an oscillating wall," Journal of Turbulence, vol. 12, article N9, 2011.

[13] M. Skote, "Temporal and spatial transients in turbulent boundary layer flow over an oscillating wall," International Journal of Heat and Fluid Flow, vol. 38, pp. 1-12, 2012.

[14] S. Lardeau and M. A. Leschziner, "The streamwise dragreduction response of a boundary layer subjected to a sudden imposition of transverse oscillatory wall motion," Physics of Fluids, vol. 25, no. 7, Article ID 075109, 2013.

[15] M. Skote, "Comparison between spatial and temporal wall oscillations in turbulent boundary layer flows," Journal of Fluid Mechanics, vol. 730, pp. 273-294, 2013.

[16] M. Skote, "Turbulent boundary layer flow subject to streamwise oscillation of spanwise wall-velocity," Physics of Fluids, vol. 23, no. 8, Article ID 081703, 2011.

[17] C. Viotti, M. Quadrio, and P. Luchini, "Streamwise oscillation of spanwise velocity at the wall of a channel for turbulent drag reduction," Physics of Fluids, vol. 21, no. 11, Article ID 115109, 2009.

[18] M. Quadrio, P. Ricco, and C. Viotti, "Streamwise-travelling waves of spanwise wall velocity for turbulent drag reduction," Journal of Fluid Mechanics, vol. 627, pp. 161-178, 2009.

[19] M. Skote, "Scaling of the velocity profile in strongly drag reduced turbulent flows over an oscillating wall," International Journal of Heat and Fluid Flow, vol. 50, pp. 352-358, 2014.

[20] A. Cimarelli, B. Frohnapfel, Y. Hasegawa, E. De Angelis, and M. Quadrio, "Prediction of turbulence control for arbitrary periodic spanwise wall movement," Physics of Fluids, vol. 25, no. 7, Article ID 075102, 2013.

[21] M. Chevalier, P. Schlatter, A. Lundbladh, and D. S. Henningson, "Simson-a pseudo-spectral solver for incompressible boundary layer flows," Tech. Rep. TRITA-MEK 2007:07, KTH Mechanics, Stockholm, Sweden, 2007.

[22] H. Schlichting, Boundary Layer Theory, McGraw-Hill, New York, NY, USA, 7th edition, 1979.

[23] F. P. Bertolotti, T. Herbert, and P. R. Spalart, "Linear and nonlinear stability of the Blasius boundary layer," Journal of Fluid Mechanics, vol. 242, pp. 441-474, 1992. 


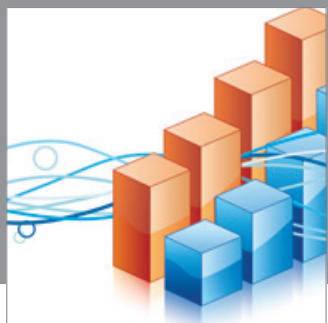

Advances in

Operations Research

mansans

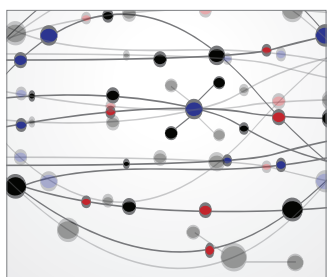

The Scientific World Journal
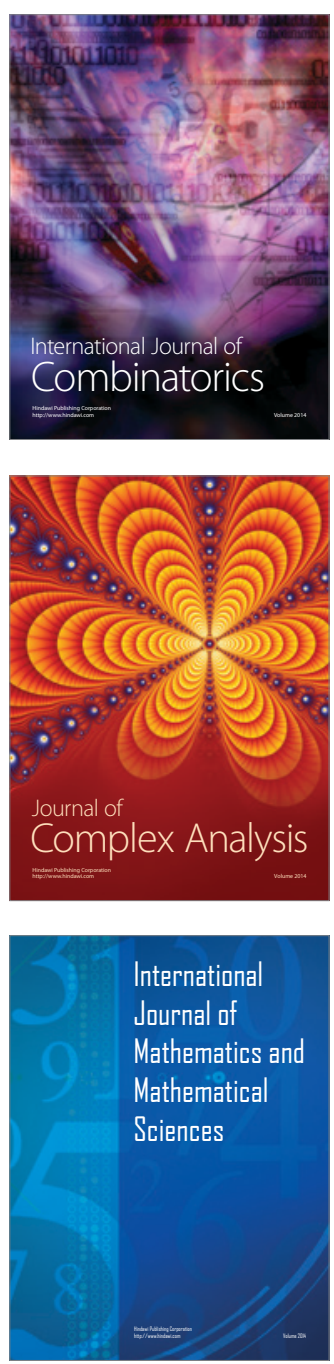
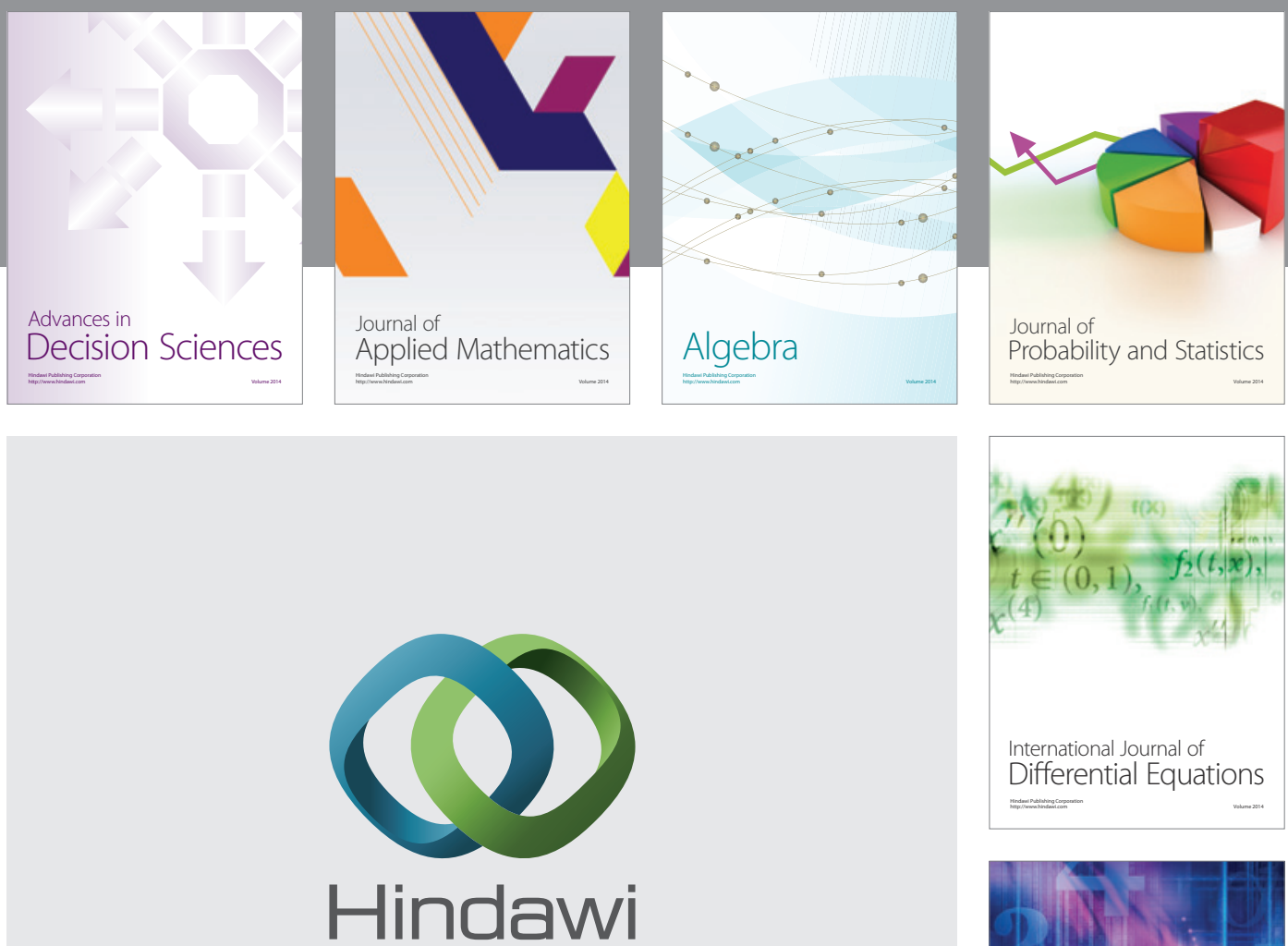

Submit your manuscripts at http://www.hindawi.com
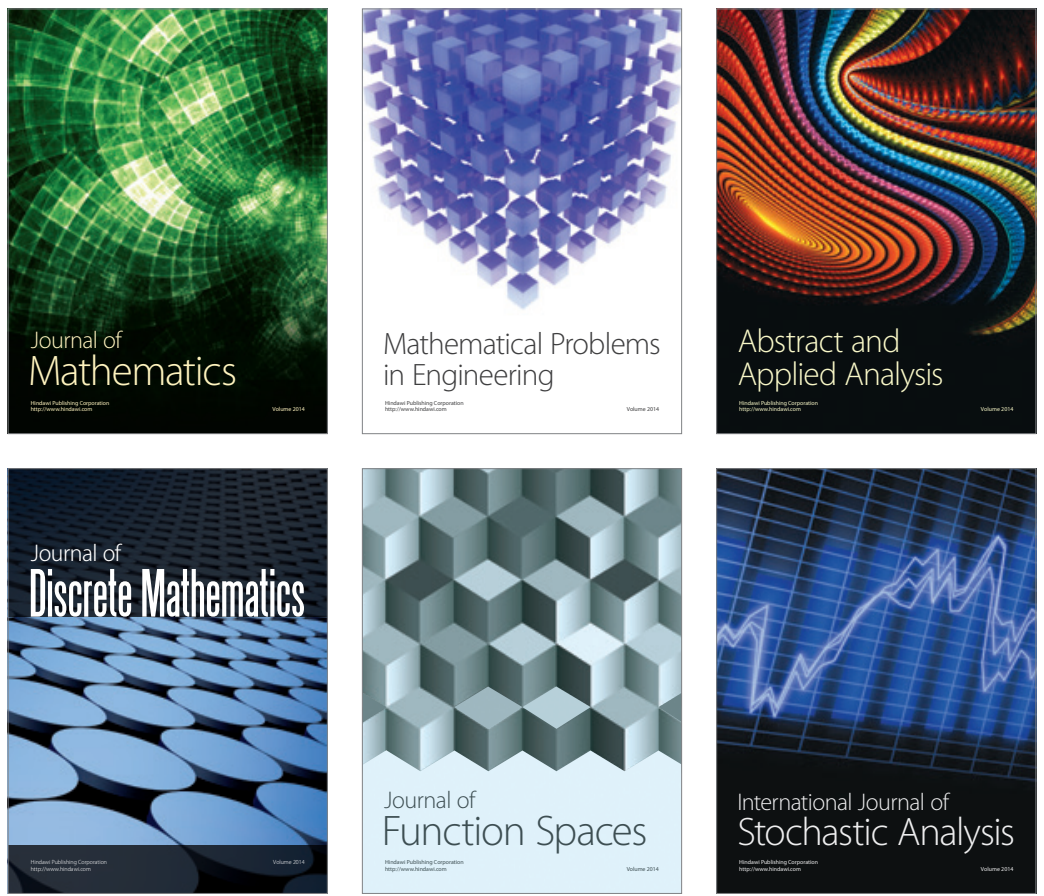

Journal of

Function Spaces

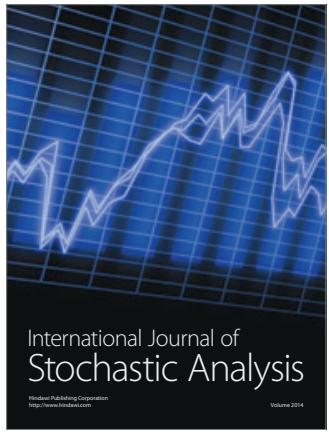

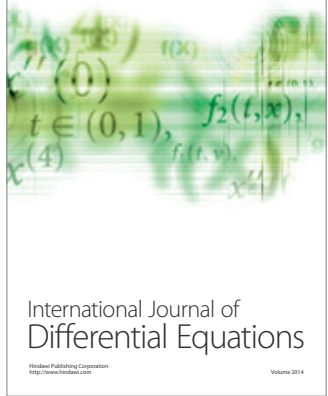
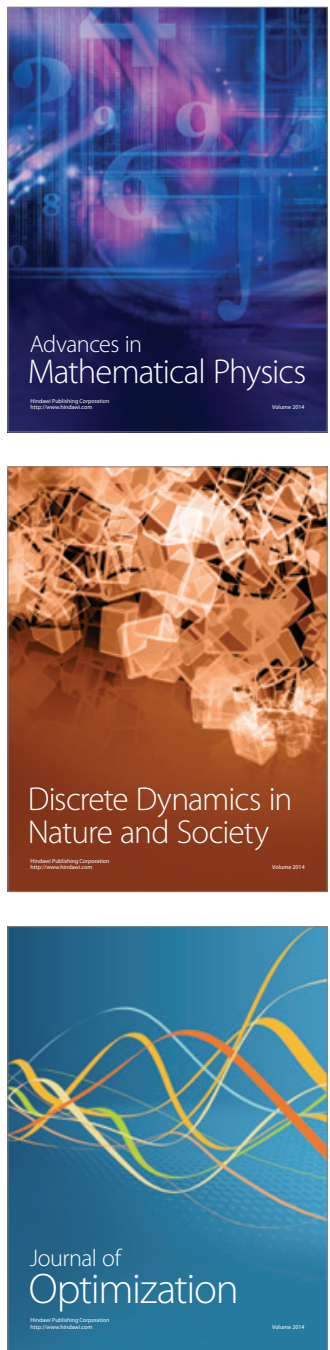